\title{
Quantifying excavation-induced rock mass damage in large open pits
}

\author{
L Lorig Itasca Consulting Group, Inc., USA \\ D Potyondy Itasca Consulting Group, Inc., USA \\ Varun Itasca Consulting Group, Inc., USA
}

\begin{abstract}
Open pit excavation induces rock mass damage as a result of stress release and blasting. The damage reduces the rock mass strength and stiffness. The strength reduction is typically quantified by the Hoek-Brown disturbance factor $(D)$. Limitations of this approach are outlined in the paper. It is proposed that degradation of rock mass strength and stiffness results from damage followed by disturbance. Damage is caused by fracturing of intact rock (e.g. failure of rock bridges) that results from small strains (less than approximately 2 percent) and only increases porosity slightly. Disturbance is caused by rearrangement of intact rock blocks that results from large strains (perhaps 10 percent) and usually produces a large increase in porosity. The strength degradation produced by damage is quantified by the peak and post-peak strengths, and the strength degradation produced by disturbance is quantified by the residual strength. When using numerical models to study the progressive failure of rock masses, estimates of the damage characteristics are required. In many of these models, the Hoek-Brown failure criterion is treated as a yield criterion and the analysis is carried out using plasticity theory. The work described here is aimed at defining the appropriate evolution of rock mass strength as applied to slope stability studies. We do this by studying the stress-strain response of $3 D$ bonded block models with low-porosity structures that range from blocky to disintegrated and a high-porosity structure that mimics rockfill. The synthetic peak and post-peak strength envelopes are compared with Hoek-Brown strength envelopes with Dfactors of zero and one. The comparisons support the following conclusions. Peak and post-peak strength are similar for lower quality (Geological Strength Index less than approximately 40) rock masses. Hoek-Brown strengths for $D=1$ are lower than post-peak strengths, with the difference being more pronounced for lower quality rock masses. For low quality rock masses, the Hoek-Brown strength for $D=1$ may be lower than the residual strength. In the Hoek-Brown approach, as the Dfactor increases, the behaviour is cohesion weakening friction weakening; however, our work suggests that the behaviour for higher quality rock masses is cohesion weakening friction strengthening. This statement applies to the synthetic material, and laboratory evidence suggests that it also applies to real rock masses.
\end{abstract}

Keywords: Hoek-Brown criterion, bonded block model, slope stability

\section{Introduction}

Stability analysis of large slopes requires estimates of rock mass strength. Rock mass strength and stiffness are reduced by deformation caused by blast damage and stress release from slope excavation. This degradation is commonly referred to as damage and/or disturbance in Hoek-Brown terms but is not easily quantifiable. Material properties (e.g. strength, stiffness) do not change significantly during the first (pre-peak) and last (residual) phases of deformation, whereas the middle phases between the onset of yield and the attainment of residual strength are associated with changing material properties as the rock undergoes progressive deformation. Accumulated plastic shear strain is a common metric for irreversible strain in geomaterials and allows damage to be quantified. As plastic shear strain accumulates, the rock mass strength goes from peak to residual.

Residual strength has no universally accepted definition. In this study, we adopt the approach commonly applied in laboratory testing studies and use the post-peak strength for comparison purposes. The post-peak 
strength is determined as the stress level at which the post-peak stress becomes approximately constant following the initial loss of strength. This paper also suggests mechanical definitions of damage and disturbance and links these to the three strengths denoted in Figure 1. The strength degradation produced by damage is quantified by the peak and post-peak strengths, and the strength degradation produced by disturbance is quantified by the residual strength.

The objective of the work summarised in this paper is to define the appropriate evolution of rock mass strength as applied to slope stability studies (Varun et al. 2019). This is done by studying the stress-strain response of synthetic rock masses under confined and unconfined axial compression. The models provide peak and post-peak strengths of rock masses with low-porosity structures that range from blocky to disintegrated and a high-porosity structure that mimics rockfill. The synthetic peak and post-peak strength envelopes are compared with Hoek-Brown strength envelopes with $D$ factors of zero and one. Numerical results are compared to Hoek-Brown strengths at low confining stresses ( $<4 \mathrm{MPa}$ ) where failures typically occur in open pit slopes.

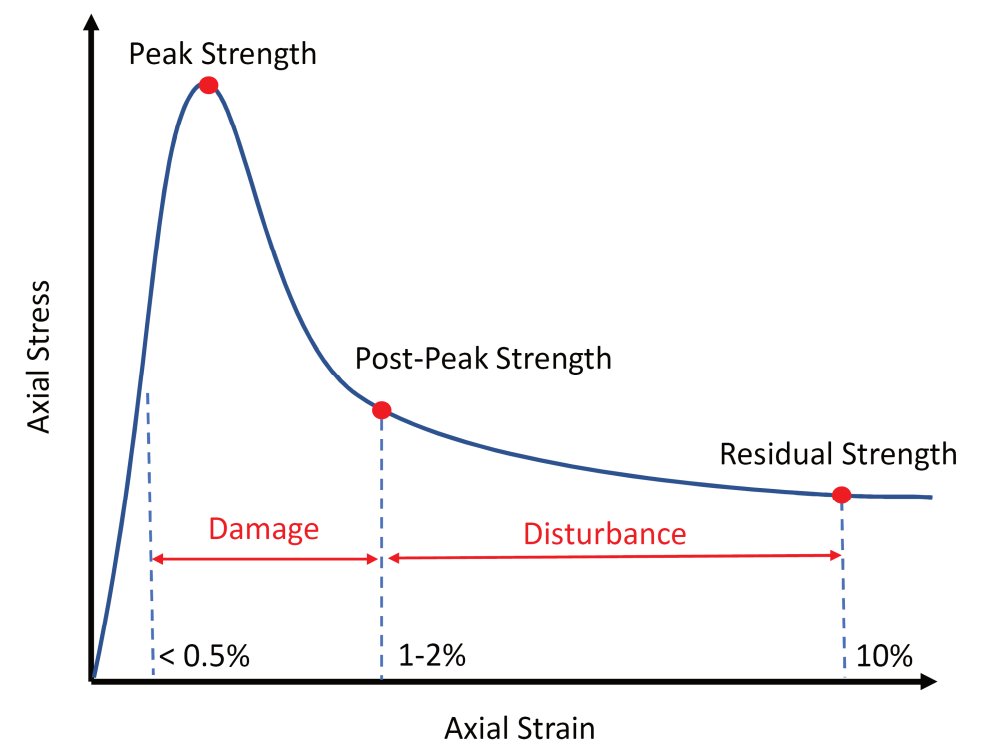

Figure 1 Conceptual stress-strain curve showing different strengths and strength degradation regimes

\section{Generalised Hoek-Brown criterion}

The generalised Hoek-Brown criterion for the estimation of rock mass strength, introduced by Hoek (1994) and Hoek et al. (1995), is expressed as:

$$
\sigma_{1}=\sigma_{3}+\sigma_{c i}\left(m_{b} \frac{\sigma_{3}}{\sigma_{c i}}+s\right)^{a}
$$

where $m_{b}, s$, and $a$ are the rock mass material constants, given by:

$$
\begin{aligned}
& m_{b}=m_{i} \exp [(G S I-100) /(28-14 D)] \\
& s=\exp [(G S I-100) /(9-3 D)] \\
& a=1 / 2+1 / 6\left(e^{-G S I / 15}-e^{-20 / 3}\right)
\end{aligned}
$$

where, for intact rock, the material constants are denoted by $m_{i}, s=1$ and $a=0.5$; and $D$ is a parameter that depends upon the degree of disturbance to which the rock mass has been subjected by stress release and blasting. The equations above were developed to deal with rock masses comprised of interlocking angular blocks under low to moderate confining stresses, for which the failure process is dominated by block sliding and rotation without a great deal of intact rock failure. The $D$ parameter in the Hoek-Brown criterion 
degrades both the cohesion and friction of the rock mass and is unbounded with respect to confinement. The $D$ parameter creates a new weaker material, as illustrated in Figure 2.

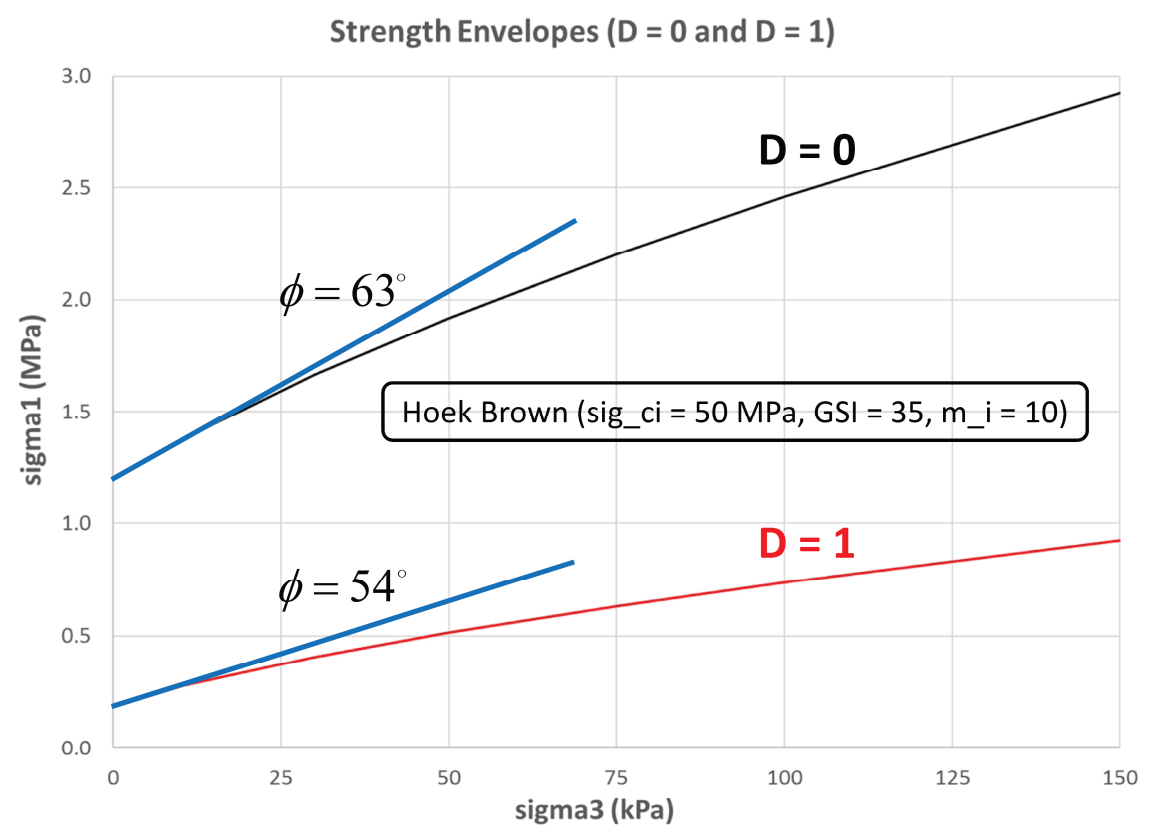

Figure 2 Typical strength envelopes for undisturbed $(D=0)$ and disturbed $(D=1)$ rock mass via the Hoek-Brown damage factor

It is important to note the following points about the Hoek-Brown approach:

1. The Hoek-Brown approach uses both damage and disturbance without rigorously defining either. Practitioners refer to the $D$ parameter as either the disturbance factor or the damage factor. Additional confusion sometimes results from the use of 'disturbance' to characterise rock mass structure within the table for selecting Geological Strength Index (GSI), as shown in Figure 3.

2. While there are guidelines for selecting the $D$ parameter (for example, see Section 8 of Hoek \& Brown 2019). The guidelines are somewhat vague and are often interpreted differently by practitioners.

3. As $D$ increases, the behaviour is cohesion weakening friction weakening; however, the results summarised in Figure 4 support the assertion that the behaviour is cohesion weakening friction strengthening, at least for higher quality rock masses (Riahi et al. 2020).

4. Poor quality rock masses (GSI less than approximately 40) may already be fully damaged and probably cannot be further damaged.

5. Experience and logic suggest that the damage caused by stress release and blasting is limited and should be made a function of confining stress (or depth for slope stability problems). Rose et al. (2018) have addressed this issue by proposing a disturbance rating for open pit mine slopes that can provide guidelines for the selection of the depth of the fully damaged conditions behind the slope and the decrease in the damage factor over a range of slope heights. This approach assumes, however, that the fully damaged strength envelope is a valid representation of the post-peak strength at low confining stresses.

6. The Hoek-Brown failure envelope represents a peak strength. For many engineering analyses, we are also interested in the residual strength. Note that $D=1$ does not necessarily correspond to a residual strength; instead, it is the peak strength of a rock mass that has been degraded by stress release and blasting. 


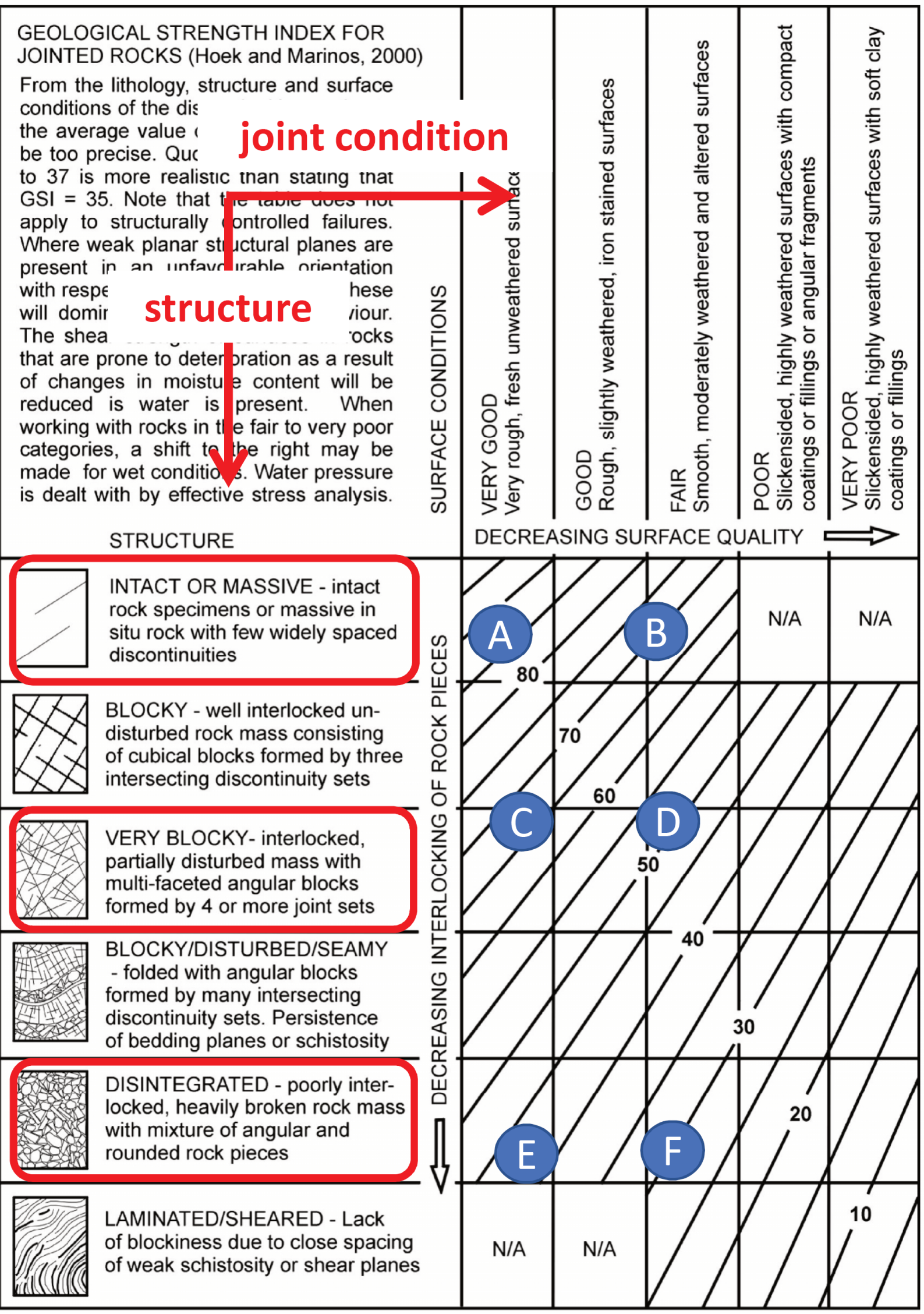

Figure 3 Basic Geological Strength Index (GSI) chart (Hoek \& Marinos 2000) along with equivalent GSI values based on structure and joint condition for the six synthetic rock mass samples 


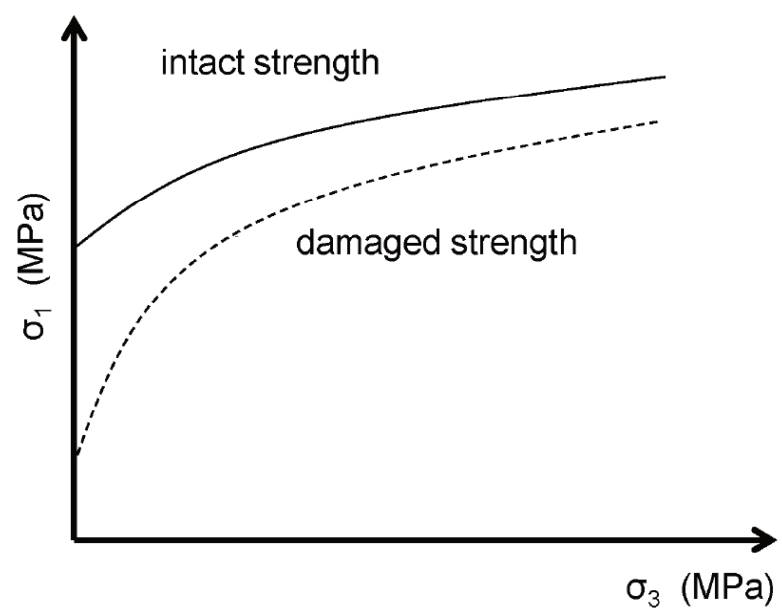

Figure 4 Schematic representation of strength envelopes for intact and damaged rock. The damaged strength characterises physical models of jointed rock masses and damaged intact rock that are considered as analogues for isotropic jointed rock masses (from Bahrani 2015)

\section{Damage and disturbance defined}

Degradation of rock mass strength results from damage followed by disturbance. For the work described here, we adopt the following definitions.

Damage is caused by fracturing of intact rock (e.g. failure of rock bridges). Damage typically results from small strains (less than approximately 2 percent) and only increases porosity slightly. At low confinement, the yield is related to creation of local extension cracks in the $\sigma_{1}$ direction. These cracks may link up in an en-échelon fashion to form through-going fractures that do not necessarily involve classical sliding. At higher confinement, these through-going fractures experience relative shear displacement and are denoted as shear bands. The Hoek-Brown failure criterion is strictly applicable to this type of small-strain induced damage, i.e. brittle shear failure that occurs at confinements below the brittle-ductile transition.

Disturbance is caused by rearrangement of intact rock blocks. Disturbance typically results from large strains (perhaps 10 percent) and produces an increase in porosity. Any changes in friction coefficient seem to be related to changes in mode of deformation. The apparent slope of the yield surface (due to micro-cracks, as noted above) changes to a slope (in $\sigma_{1}-\sigma_{3}$ space) that is more related to actual sliding on surfaces that are angled with respect to the $\sigma_{1}$ direction. This sliding may involve slip of point contacts on planes, or slip on through-going bumpy surfaces, or combinations of both. Thus, the friction coefficient may go up or down (with respect to its value in the low-confinement region), depending on the geometry and nature of the shearing surfaces.

\section{$4 \quad$ Bonded block modelling}

Continuum numerical modelling is inherently limited when the rock behaviour involves mechanisms such as spalling and bulking. The Bonded Block Model (BBM) approach allows simulation of the initiation of cracks that can coalesce and/or propagate, leading to extension and shear fracturing, and thereby inducing a strength dependency on confinement for rock that may initially be intact, jointed, or veined (Garza-Cruz \& Pierce 2014). BBMs represent a solid as a bonded collection of polyhedral blocks. Such models provide a synthetic material with mechanical behaviour ranging from that of a solid material (such as rock) when the bonds are intact, to that of a tightly interlocked granular material when the bonds have all broken. These models provide a wide range of rock behaviours at both an intact and rock mass scale. The mechanical behaviour of these models is simulated by the distinct-element method using the 3DEC code, in which the blocks are rigid or deformable polyhedra. The blocks can be tetrahedral or Voronoi-shaped polyhedra. This 
study uses tetrahedral blocks because they allow more pathways for a fracture to propagate, are less prone to numerical stability issues, and are easier to implement (Azocar 2016).

A calibration is performed where contact stiffness, tensile strength, and cohesion are adjusted to match the Young's modulus, macroscale tensile strength, and unconfined compressive strength of the intact rock. The friction angle between initially bonded contacts and between new contacts that form as a result of additional deformation is kept constant at 30 degrees for most cases except where noted otherwise.

\subsection{Boundary conditions}

Direct tension, uniaxial compressive strength (UCS), and triaxial tests are performed. The tests are all conducted on a cylindrical specimen of 2:1 aspect ratio, which is similar to a typical lab specimen. The confining pressure for triaxial tests is applied using a pressure boundary condition on the outer faces of blocks.

\subsection{Rigid versus deformable blocks and block plasticity}

Rigid blocks are used for the initial simulations. The blocks can be made deformable and plasticity can also be added, but this adds computational overhead. For deformable blocks, the compliance must be distributed between the blocks and the contacts. Although concentrating the compliance in the blocks allows the Poisson's ratio for an intact rock to be matched, concentrating the compliance in the contacts is preferred for the following reasons:

- Concentrating the compliance in the blocks causes the post-peak response to evolve at a slower rate (i.e. intact hard rock specimens are less brittle) than for models in which the compliance is concentrated in the contacts. Preliminary analyses suggest that this is because damage in the rock is proportional to the magnitude of displacements at the contacts. With more compliant blocks, most of the displacement goes into the blocks, and as a result, larger axial displacements (strains) are required to induce the same amount of damage as for the case when compliance is concentrated in the contacts.

- Concentrating the compliance in the contacts produces softening behaviour that is like that of rigid blocks and allows for comparison of results obtained from models using block plasticity with the rest of the samples using rigid blocks.

The stress-strain response for a UCS test for both cases is shown in Figure 5. Note the slow rate of reduction in axial load for the case with compliance concentrated in the blocks.

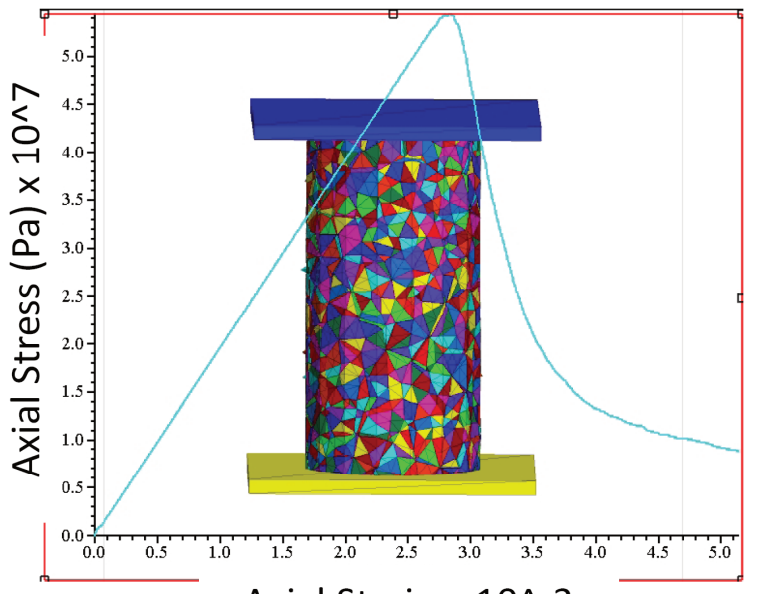

Axial Strain $\times 10^{\wedge}-3$

(a)

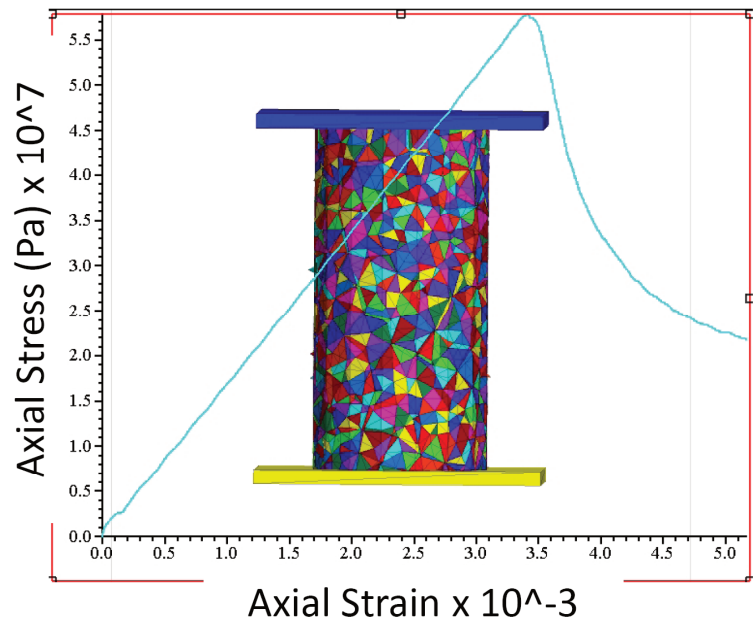

(b)

Figure 5 Axial stress-strain response for model with compliance concentrated (a) in the contacts and (b) in the blocks 
Unless the plasticity in the blocks is important (discussed below), rigid blocks are able to capture the rock behaviour and deformable blocks are not needed. For cases where block plasticity is important, deformable blocks with compliance concentrated in the contacts are used, because such models have the same pre-peak stress-strain response as rigid blocks.

The blocks in the BBM do not represent rock grains; instead, they represent rock fragments with boundaries along which fractures can propagate. The strength of each block is thus the same as that of a piece of intact rock. However, when rigid blocks are subjected to large strains, they can become locked together and will carry huge forces that a fragment of the same size in a real sample would not be able to sustain. In a real sample, the fragment would break down further. Tests on rockfill (Xiao et al. 2015) indicate that the percentage of fragments that break during loading increases as a function of confining stress.

To evaluate the effect of unbreakable blocks, BBM models for intact rock are created using both rigid and plastic blocks, and then subjected to triaxial tests with varying confinement. Confinement ranging from zero to four MPa spans the range of interest for confinement of open pit slopes, because rock subjected to larger confinement is farther away from the slope face and not expected to be involved in failure. The response from the two models is comparable for low confinement (0 to $4 \mathrm{MPa})$, and therefore, the rigid-block assumption is adequate for such low confinement. For higher confinement (above $4 \mathrm{MPa}$ ), the rigid blocks exhibit an unrealistic hardening-type behaviour at larger strains (greater than one percent). However, samples of lower quality rock masses exhibit unrealistic hardening behaviour at confining stresses less than $4 \mathrm{MPa}$ as well. Therefore, rigid blocks are used only for higher quality samples (GSI > 80), and plastic blocks are used for the rest of the simulations.

\section{$5 \quad$ Numerical samples}

All bonded block samples are generated by modifying a base configuration with a sample height of $12 \mathrm{~m}$ and a sample diameter of $6 \mathrm{~m}$. The sample is discretised using tetrahedral blocks with sizes ranging from 0.75 to $1 \mathrm{~m}$. The base configuration is calibrated to match the stiffness and strength properties of typical intact rock. The macro properties of the intact rock are Young's modulus of $20 \mathrm{GPa}$, tensile strength of $3.3 \mathrm{MPa}$, and UCS of $53 \mathrm{MPa}$. The response of the base configuration during a UCS test is shown in Figure 6.

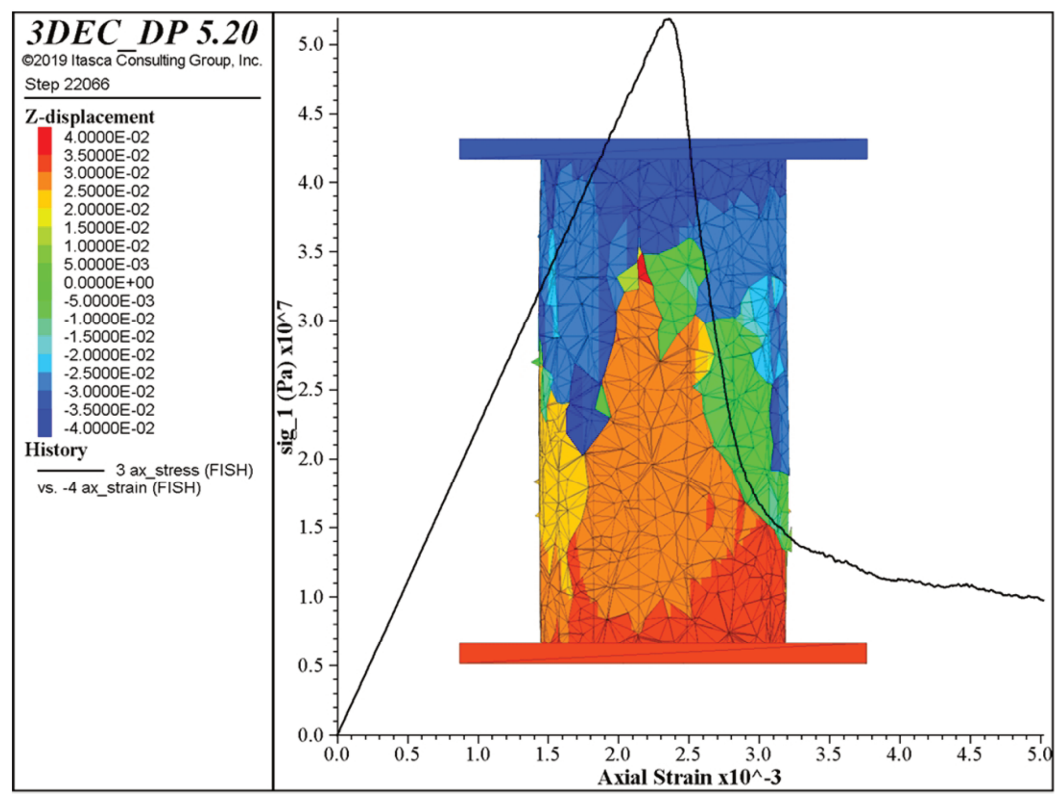

Figure 6 Axial stress-strain response for a uniaxial compressive strength test performed on the base configuration. The vertical displacement contours indicate a spalling-type failure 


\subsection{Rock mass models}

The rock mass models consist of two high quality and four low quality rock masses and are shown in Figure 7. A high quality rock mass is modeled by adding a discrete fracture network (DFN) to the base configuration. DFNs can be added to BBMs by cutting the blocks, or blocks can be generated with DFNs as part of the boundaries. The block-cutting approach is used for this study. Even though the same specimen is used as the starting point as for intact rock, cutting in the DFN changes the microstructure of the blocks. We no longer have all tetrahedral blocks; hence, the calibration between contact properties and macro properties changes. For these cases, to simulate the intact rock specimen for comparison with the rock mass, the DFN joints are assigned the same strength as the bonded contacts, and the intact rock UCS is determined again. An intact rock UCS of $32 \mathrm{MPa}$ and a tensile strength of $2 \mathrm{MPa}$ is obtained. The following two samples are set up using this method:

- Sample A: The sample consists of three orthogonal joint sets with a fracture frequency P10 =0.5/m for each set. Three joint sets are chosen to reduce any anisotropy in strength response as a function of loading angle. The joint sets are oriented such that no set is aligned with the loading direction. The friction angle for the DFN joints is $\phi=35^{\circ}$, and for any new contacts formed by the breaking of existing contacts, $\phi=30^{\circ}$.

- Sample B: The sample consists of three orthogonal joint sets with a fracture frequency P10 $=0.5 / \mathrm{m}$ for each set like sample $A$. The friction angle for the DFN joints is $\phi=20^{\circ}$, and for any new contacts formed by the breaking of existing contacts, $\phi=20^{\circ}$.

A low quality rock mass is modeled by randomly breaking a certain percentage of contacts in the base configuration. The following four samples are set up using this method:

- Sample C: $60 \%$ of the initial contacts are broken. The broken contacts and any new contacts are assigned a friction angle of $\phi=30^{\circ}$.

- Sample D: $60 \%$ of the initial contacts are broken. The broken contacts and any new contacts are assigned a friction angle of $\phi=20^{\circ}$ to represent a relatively poor joint condition.

- Sample E: $100 \%$ of the initial contacts are broken to represent a fully damaged sample but still interlocked with almost zero porosity. The broken contacts and any new contacts are assigned a friction angle of $\phi=30^{\circ}$.

- Sample F: $100 \%$ of the initial contacts are broken. The broken contacts and any new contacts are assigned a friction angle of $\phi=20^{\circ}$.

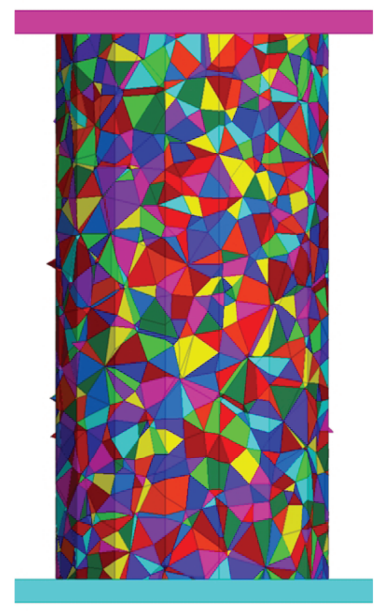

(a)

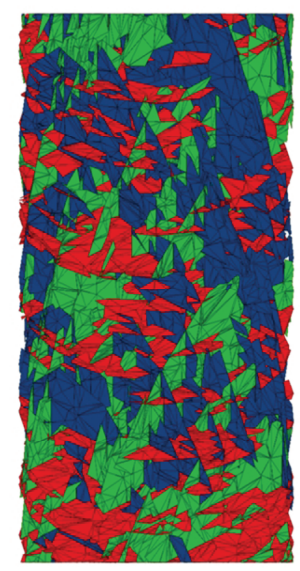

(b)

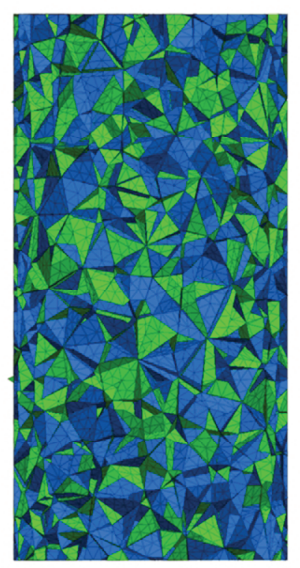

(c)

Figure 7 (a) Base configuration for intact rock; (b) DFN joints cut in for samples A and B; (c) Broken and intact contacts for samples $C$ and $D$ 


\subsection{Rockfill model}

A fully disturbed rock mass is modelled as rockfill. The sample is created by packing the same blocks used in the base configuration under gravity in a container of the same radius but $16 \mathrm{~m}$ (instead of $12 \mathrm{~m}$ ) high. Then blocks with centroids above $12.25 \mathrm{~m}$ height are deleted, and the top platen is moved down to create a sample $12 \mathrm{~m}$ high. Confinement is applied by using rigid side walls moving radially until the average stress on the walls reaches the desired confining stress. The blocks are exported to a new model and confined using a flexible sleeve. Axial load is applied by moving both top and bottom platens. The contacts have a friction angle of $\phi=30^{\circ}$. A comparison between the near-zero porosity base configuration and the $40 \%$ porosity rockfill model is shown in Figure 8.

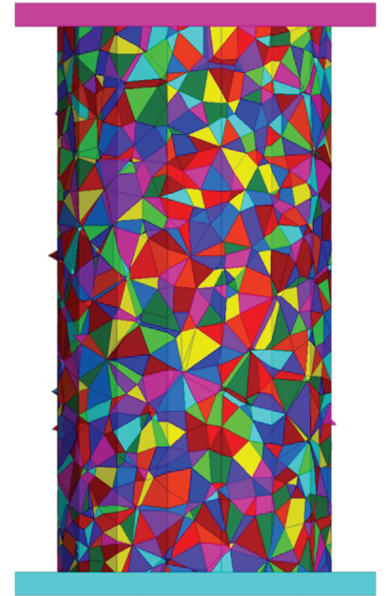

(a)

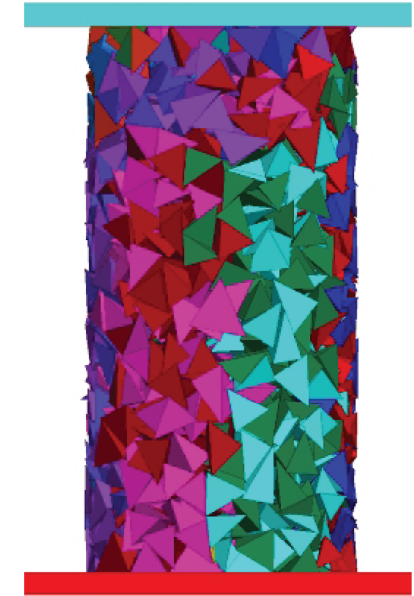

(b)

Figure 8 (a) Intact rock with almost zero porosity; (b) Gravity packed rockfill with $40 \%$ porosity

\section{$6 \quad$ Results}

The axial stress-strain curves for the six samples are shown in Figure 9. The peak and post-peak strengths are determined based on these stress-strain curves, and the strength envelopes are shown in Figure 10. The stress-strain curves use engineering stress (total axial force divided by initial cross-sectional area of the sample). However, the peak strength for samples $E$ and $F$ is mobilised at strains greater than $5 \%$, at which point there is a significant difference between the initial and final cross-sectional areas. To account for this difference, the strength envelopes for samples $E$ and $F$ are determined by correcting for the change in cross-sectional area. Except for UCS, the peak and post-peak strengths are equal for samples $E$ and $F$.

Using the intact UCS values, Hoek-Brown failure envelopes for $D=0$ are fitted to peak strength envelopes and equivalent GSI values are determined (see Table 1). Corresponding Hoek-Brown failure envelopes for the $D=1$ case are then determined using the fitted GSI values and plotted for comparison. Based on the GSI values and joint structure and condition, the samples are also plotted on the GSI chart shown in Figure 3. 

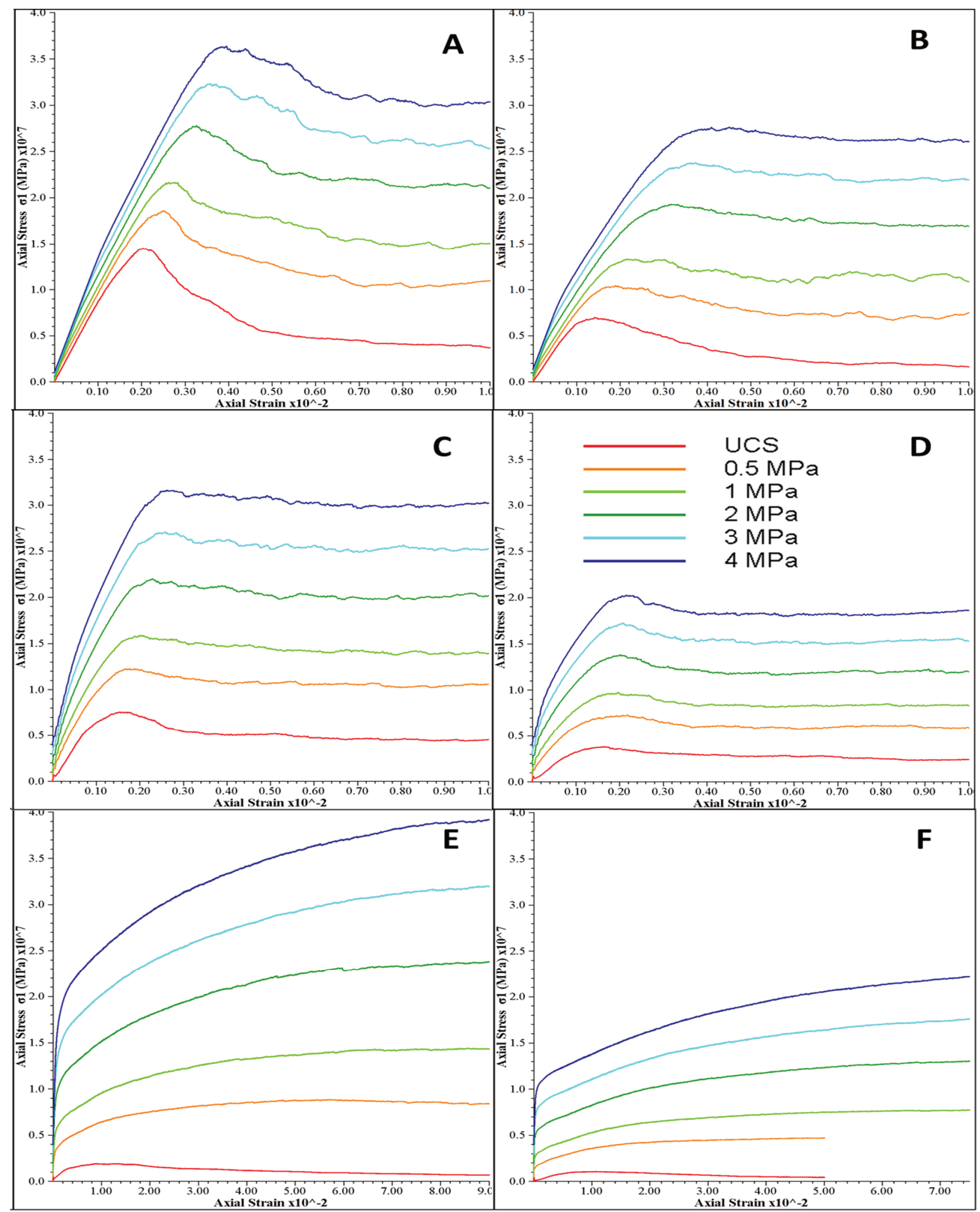

Figure 9 Axial stress-strain curves of the six samples for confinements ranging from 0 to $4 \mathrm{MPa}$ 

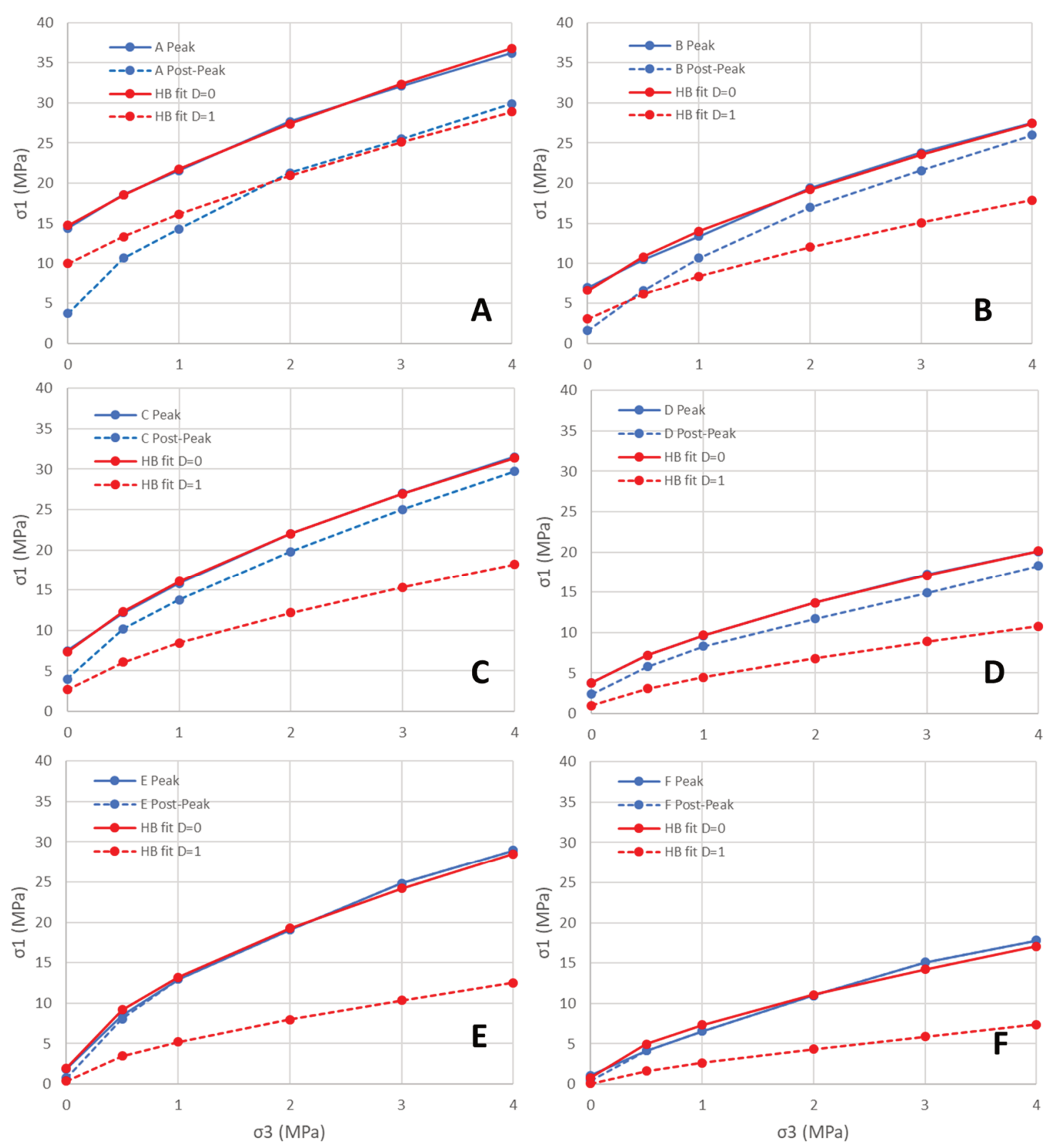

Figure 10 Peak and post-peak strength envelopes of the six samples along with fitted Hoek-Brown strength envelopes

Table 1 Fitted Geological Strength Index (GSI) and $m_{i}$ values for the six samples described in Section 5.1

\begin{tabular}{lllllll}
\hline Sample & A & B & C & D & E & F \\
\hline$m_{i}$ & 11.0 & 10.8 & 11.6 & 6.3 & 23 & 12 \\
GSI & 86 & 71 & 64 & 52 & 42 & 28 \\
\hline
\end{tabular}

The model response is summarised and compared with the Hoek-Brown response as follows:

- Effect of confinement: As confinement increases, the stiffness, peak strength, and post-peak strength increase for all six samples. The stress-strain response transitions from brittle to ductile, and the difference between peak and post-peak strength decreases as confinement increases. The axial strain required to reach peak strength also increases as confinement increases. Although it is 
difficult to determine precisely the plastic strain increment between peak and post-peak strength, a qualitative comparison indicates that the plastic strain increment for each sample does not change significantly due to confining stress in this range. At low confining stresses, the strength envelopes exhibit cohesion weakening and friction strengthening for all samples except for samples $\mathrm{D}$ and $\mathrm{F}$, which show almost the same friction. As confinement increases, the post-peak strength envelopes become almost parallel to the peak strength envelopes.

- Effect of rock quality (GSI): As GSI decreases, the peak and post-peak strengths decrease as expected. The stress-strain response is also more ductile for the lower quality (lower GSI) rock masses as compared to a more brittle response for the higher quality rock masses. Samples E and F with $100 \%$ contacts broken show very ductile behaviour, with axial strains of $1-10 \%$ required to reach peak strength, whereas samples $A$ to $D$ reached peak strengths in $<0.5 \%$ strain. The difference between peak and post-peak strength decreases as rock mass GSI decreases, i.e. lower quality rock masses have less potential for further damage. The plastic strain increment from peak to post-peak strength also decreases as rock mass quality decreases, with no difference between peak and postpeak strength for samples $E$ and $F$. This makes sense based on our proposed definition of damage, because a high quality rock mass has more potential for damage compared to a low quality rock mass. If damage is related to plastic strain increment, then one would expect larger plastic strain increments between peak to post-peak transition for a higher quality rock mass as observed here. Hoek-Brown strengths for $D=1$ are lower than post-peak strengths for most samples. The difference is more pronounced in lower quality rock masses where Hoek-Brown predicts a larger strength reduction for $D=1$, which is counter-intuitive. Intuitively, we would expect lower quality rock masses to have a lower strength reduction, because they are already highly damaged, and there is little potential for more damage.

The comparison between failure envelopes for sample $E$ and the rockfill sample is shown in Figure 11. Both samples have a contact friction of $30^{\circ}$ and are completely damaged (i.e. there are no intact bonds), but the difference is in terms of disturbance, with almost zero porosity for sample $E$, whereas the rockfill sample has approximately $40 \%$ porosity. The strength envelope for the rockfill can be thought of as the residual strength envelope for sample $\mathrm{E}$. The results show lower strength for the rockfill sample as expected when compared with sample E. However, the $D=1$ Hoek-Brown strength envelope falls below that of the rockfill in this case.

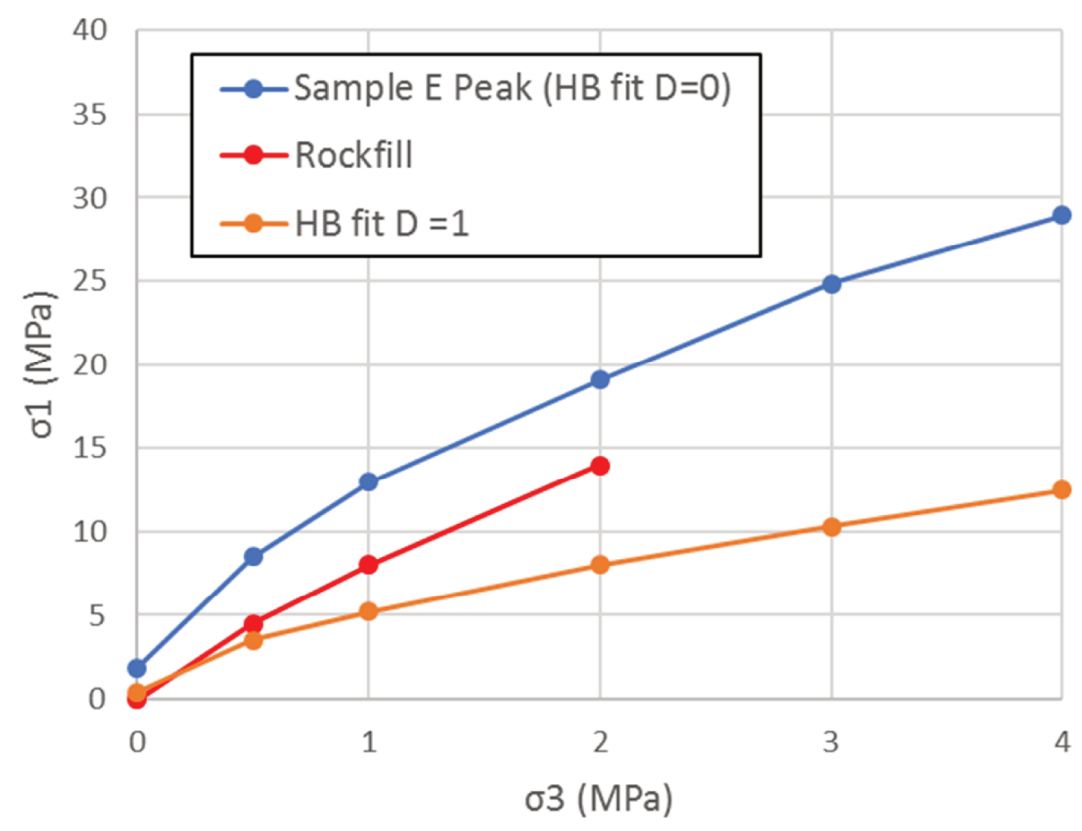

Figure 11 Comparison between peak strength envelopes for Sample E and rockfill sample 


\section{$7 \quad$ Conclusions}

This study resulted in the following conclusions:

- Peak and post-peak strengths are similar for lower quality $(\mathrm{GSI}<\sim 40)$ rock masses. More work is required to better define this limit.

- Hoek-Brown strengths for $D=1$ are lower than post-peak strengths for most samples. The difference is more pronounced in lower quality rock masses where Hoek-Brown predicts a larger strength reduction for $\mathrm{D}=1$, which is counter-intuitive. Intuitively, we would expect lower quality rock masses to have a lower strength reduction, because they are already highly damaged, and there is little potential for more damage.

- For low quality rock masses, the $\mathrm{D}=1$ strength may be lower than the residual strength.

- The post-peak strength envelopes exhibit cohesion weakening and friction strengthening behaviour at low confinement for all samples except samples $D$ and $F$, which show almost the same friction. As confinement increases, the peak and post-peak strength envelopes become almost parallel.

- Accumulated plastic shear strain is a common metric for irreversible strain in geomaterials and allows damage to be quantified. All that remains before introducing the failure envelope results into the Itasca constitutive Model for Advanced Strain Softening (IMASS) is converting the total axial strains in the numerical model to plastic shear strains. There should be no difficulties in doing this. Once this is done, the constitutive relation can be used in any numerical model to simulate the rock mass strength evolution and validate the results with case histories.

\section{Acknowledgement}

The work described here was performed under a Collaborative Research Agreement with the University of Queensland in Brisbane, Australia as part of the Large Open Pit Project. The sponsors of this project are thanked for their support. Significant contributions to this work by Itasca colleagues-Peter Cundall, Matt Purvance and Azadeh Riahi-are also gratefully acknowledged. The authors also thank the anonymous reviewers and Dr Evert Hoek for their constructive comments and suggestions.

\section{References}

Azocar, K 2016, Investigating the mesh dependency and upscaling of 3D grain-based models for the simulation of brittle fracture processes in low-porosity crystalline rock, MSc Thesis, Queens University, Kingston.

Bahrani, N 2015, Estimation of confined peak strength for highly interlocked jointed rockmasses, PhD Thesis, Laurentian University, Sudbury.

Garza-Cruz, TV \& Pierce, M 2014, 'A 3DEC model for heavily veined massive rock masses', Proceedings of the 48th U.S. Rock Mechanics/Geomechanics Symposium, American Rock Mechanics Association, Alexandria.

Hoek, E 1994, 'Strength of rock and rock masses,' ISRM News Journal, vol. 2, no. 2, pp. 4-16.

Hoek, E, Kaiser, PK \& Bawden, WF 1995, Support of Underground Excavations in Hard Rock, AA Balkema, Rotterdam.

Hoek, E \& Marinos, PG 2000, 'Predicting tunnel squeezing problems in weak heterogeneous rock masses', Tunnels and Tunnelling International, vol. 132, no. 11, pp. 45-51.

Hoek, E \& Brown, ET 2019, 'The Hoek-Brown failure criterion and GSI - 2018 edition', Journal of Rock Mechanics and Geotechnical Engineering, vol. 11, no. 3, pp. 445-463.

Riahi, A, Potyondy, D \& Lorig, L 2020, Rock damage - A literature review, Itasca Consulting Group, Inc., Report to Large Open Pit Project, University of Queensland, Brisbane.

Rose, ND, Scholz, M, Burden, J, King, M, Maggs, C \& Havaej, M 2018, 'Quantifying transitional rock mass disturbance in open pit slopes related to mining excavation', Proceedings of the XIV International Congress on Energy and Mineral Resources, Asociación Nacional de Ingenieros de Minas, Seville, pp. 1273-1288.

Varun, Lorig, L \& Potyondy, D 2019, LOP II: Effect of rock mass damage on strength degradation in large open pits, Itasca Consulting Group, Inc., Report to Large Open Pit Project, University of Queensland, Brisbane.

Xiao, Y, Liu, H, Zhang, W, Yin, F \& Wang, Y 2015, 'Testing and modeling of rockfill materials: a review,' Journal of Rock Mechanics and Geotechnical Engineering, vol. 8, pp. 415-422. 
